\title{
A SURVEY ON CARDIAC DISEASES DETECTION USING DEEP LEARNING ON ECG SIGNALS
}

\author{
Snehal Pawar, Sakshi Joshi, Nikita Helude, Kalyani Shelar, Nilam Kadale \\ Department of Computer Engineering, \\ NBN Sinhgad School of Engineering, Ambegaon (BK) Pune
}

\begin{abstract}
One of the most common disease in the world is Cardiac Arrhythmia Disorder. It is one of the leading cause of deaths today. Many methods and tools have been developed to detect this diseases. Electrocardiogram (ECG) is one of the common tools used today. Using many electrocardiogram (ECG) signal we can detect and study many heart diseases. Arrhythmia is one such disease where heart fails to maintain the necessary flow of blood in normal conditions. This paper gives an approach to design a classifier based system for the automated detection of Arrhythmias. The most commonly used method for detection of arrhythmia is recording of ECG. The ECG diagram has the activity of the heart measured in electrical signals. The electrical characteristics of the heartbeat has proven to be an important tool for detecting Cardiac arrhythmias. The variety of types of Arrythmia, which are corresponding to the various patterns in ECG are represented with various waveforms. Thus, it considered essential to carry out monitoring as well as recognition of ECG signal. It also helps to reduce the time required and error rate, that is normally generated with manual reading of large ECG signals.
\end{abstract}

\section{Keywords-Arrhythmia, ECG, CNN, PSO, FFNN, LSTM}

\section{INTRODUCTION}

A commonly used tool for monitoring and recording heart activity is Electrocardiogram (ECG). The ordinary causes of chest pain, cardiac abnormality along with abnormal rhythm of heart can be detected with the ECG patterns. Once the ECG is recorded, it is analysed to diagnose the disease, and after the disease is confirmed, is it treated with the appropriate medical treatment. It is also necessary for doctors to accurately classify the type of Arrhythmia disease, as it helps for doctors to provide the best suitable treatment to the patient for disease curing. The classification of ECG waveforms to various types is a difficult and challenging task because of variability in normal ECG waveform of each person, dissimilar signs for one disease on different patients' ECG waveform. It is known in medical science that two different diseases can have nearly similar effects on two different patients' ECG waveforms, and non-existence of optimal classification rules for ECG beat classification. Various heart disease which are very common in most of the individuals are such as type of heart disease are heart failure, hypertensive heart disease, coronary artery disease, heart murmurs, congenital heart disease, pulmonary stenosis, cardiomyopathy, and rheumatic heart diseases are some of the heart diseases. Normal healthy hearts have a cardiac ECG shape. Any irregularity in the rhythm of heart can change the shape of ECG signal. There can be many types of abnormalities present in the ECG signal. An arrhythmia is an abnormality in the heartbeat pattern or heart rhythm, or the heartbeat can be too slow, too fast, have extra beats, or skip a beat. The "Left Bundle Branch Block" (LBBB), "Right Bundle Branch Block" (RBBB), "Premature Ventricular Contradiction" (PVC), "Atrial Premature Beats" (APB), and "Paced Beats" (PB) are different types of arrhythmia. ECG signals are formed of $\mathrm{P}$ wave, $\mathrm{T}$ wave, and QRS Complex. Each peak 'P', 'Q', 'R', 'S', 'T', 'U', each intervals 'RR', 'PR', 'ST', 'QRS', 'QT', and the segments 'ST', 'PR' of ECG signals have their specific amplitudes or duration values. These peaks, segments, and intervals are known as the features of ECG signal. These features are useful to classify ECG beats into the arrhythmia types. The figure shown below depicts such features of an ECG cardiac cycle.

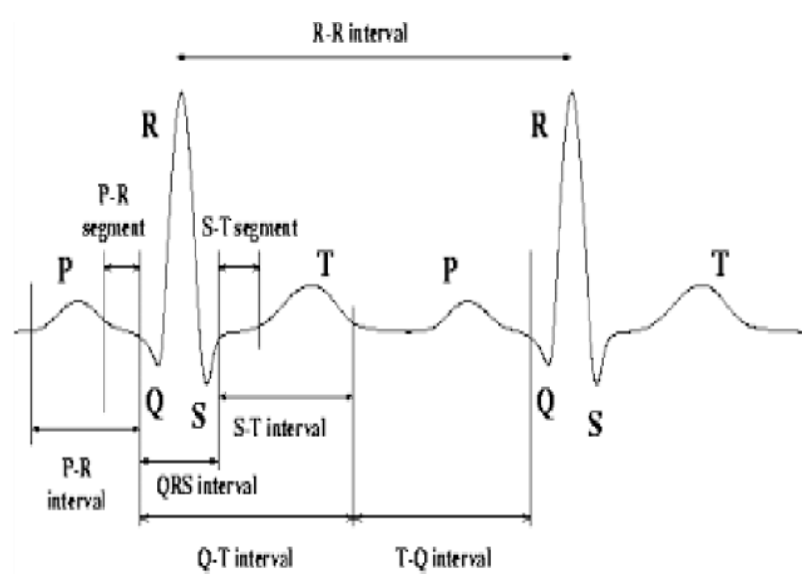




\section{International Journal of Engineering Applied Sciences and Technology, 2021 Vol. 5, Issue 11, ISSN No. 2455-2143, Pages 197-200 \\ Published Online March 2021 in IJEAST (http://www.ijeast.com)}

\section{LITERATURE SURVEY}

The paper [1] proposes a CNN model that uses the concept of time-incremental attention-based model for multiclass arrythmia disorder detection. It used ECG signals of various lengths. The data is obtained from the open source platform China Physiological Signal Challenge. It has been observed that the ATI-CNN model increased the accuracy by $7.7 \%$ on average, if compared with the older VGGNet Model. The final accuracy of the model is found to be $81.2 \%$. The future scope of the research includes the other types such as trigeminy, Twave alternans, etc.

The authors from paper [2], proposed an analysis of cardiac diagnosis using a powerful deep learning pipeline. Logistic Regression and DL pipeline's CNN (InceptionV3) has been used to classify the images in five types, from the open source Kaggle dataset MIT-BIH arrhythmia. The system is made scalable and powerful that can overcome the massive images of heart disease in big data analysis from healthcare field.

In paper [3], different cardiac diseases are diagnosed using deep learning methods such as Multilayer Perceptron network (MLP) and Convolutional neural network (CNN). The researchers build two models by using Dataset from PhysioBank.com for MLP model and Dataset from kaggle.com for $\mathrm{CNN}$ model. The result shows that proposed models MLP and CNN make efficient diagnosis of cardiac diseases with $88.7 \%$ accuracy and $83.5 \%$, respectively.

In paper [4], for diagnosis of Heart Arrhythmias, combination of frequency, morphological, and nonlinear features of ECG signal dataset are used. It is achieved by using various algorithms like NSGAII, KNN, FFNN. Among all this algorithms Feed Forward Neural Network (FFNN) have highest accuracy of $88.75 \%$ for detecting heart arrhythmia. In this model only seven class of heart arrhythmia are detected, and the remaining classes has been added in the future scope of the project.

In paper [5], ECG signals are used, and the efficient detection is carried out on congestive heart failures. The techniques that are used for evaluation involve "Quadratic Support Vector Machine" (QSVM) and "10-fold cross validation". The accuracy over $90.6 \%$ and sensitivity over $91.2 \%$ were obtained by using the above techniques on all datasets used. The research is found to be incompatible as model takes more time for training and predictions, which increases the complexity if more features are added. It is also found that the selection of appropriate kernel function is difficult in SVM. Also, the SVM model is found to be slowed down when the number of ECG signals are increased.
In paper [6], detection and classification of Cardiac Arrhythmia with 12-lead Electrocardiogram LSTM model is being used. Accuracy of LSTM model was greater than 0.9 with an area of operating characteristic curve of greater than 0.9 . Even though the model showed a very good accuracy, the records taken under consideration are as low as 116. Also, the prediction of only 12 rhythm classes is carried out in the project. The model failed to detect ST-T changes.

In paper [7], The detection of Atrial fibrillation is done using a hybrid network formed by combining CNN and LSTM models ECG dataset. Long short-term networks are used for implementation along with deep learning model. The dataset used is MIT-BIH. It has been observed that the CNN-LSTM model shows better performance even when the dataset is highly imbalanced. The proposed model predicts the overall accuracy of $89.8 \%$.

In paper [8], Heart disease prediction by neural network is done. Atrial Fibrillation database of MIT-BIH dataset is used for prediction model. R-R detection model is implemented in this paper. The mean square error observed is very low. Thus, the overall efficiency achieved is $70 \%$ whereas efficiency of the algorithm used is $80 \%$.

The paper [9] uses the combination of two technologies. These technologies are "Feed Forward Neural Network" (FFNN) and "Particle Swarm Optimisation" (PSO) for classifying ECG beats. The authors have used the database of MIT-BIH, which has collection of ECG images. This database is then differentiated into three different datasets, for checking the accuracy of all classifiers. The accuracy obtained using PSOFFNN Classifier with Pan-Tomkins algorithm is greater as compared to the "Support Vector Machine" (SVM) and "Multi- Layer perceptron Neural Network" (ML-PNN) for classification of ECG beats.

The research [10] also uses the PSO and FFNN combination, where the purpose of classification is achieved using FFNN and the weight of various parameters are achieved using the PSO optimization technique. The detection of QRS duration and heart rate is carried out using Pan Tompkins algorithm. The overall accuracy achieved of over $93 \%$ and sensitivity of around $92 \%$ were obtained by using the above techniques on dataset used.

In paper [11], the deep learning architecture has been used for the classification of ECG. The classification is divided in two classes which predicts if the patient is suffering from Arrhythmia or not. It is achieved by using various algorithms like of CNN with RNN, LSTM, GRU, etc. The accuracy was obtained on five-fold cross validation using CNN-LSTM is 0.83 for detecting heart arrhythmia. The most important advantage of the proposed method is that it does not require 


\section{International Journal of Engineering Applied Sciences and Technology, 2021 \\ Vol. 5, Issue 11, ISSN No. 2455-2143, Pages 197-200 \\ Published Online March 2021 in IJEAST (http://www.ijeast.com)}

any separate mechanism for feature engineering or noise filtration.

In paper [12], Arrhythmia Classification is done using CNN and STFT-based Spectrogram, using ECG data. This is known as $2 \mathrm{D}-\mathrm{CNN}$ model. ECG recordings are used from MIT-BIH dataset. The proposed $\mathrm{CNN}$ classifier model is validated where the manual pre-processing of ECG signals is not carried out and still the accuracy of the 2DCNN model is $87 \%$.

The paper [13], uses a simple deep learning algorithm. The technique of Convolution Neural Network is used that can efficiently classify signals. It is found from the experiments that the ELU activation function increased the accuracy of the model to $93.6 \%$. From the study of paper, it was summarised that only seven class of arrhythmia is used for the prediction.

\section{ECG DATASET}

The ECG signals are being processed using MIT-BIH dataset. The MIT-BIH Atrial Fibrillation dataset is made up of five main classes $-\mathrm{APB}, \mathrm{LBBB}, \mathrm{PVC}, \mathrm{N}$, and RBBB. The peculiar characteristic of the BIDMC $(\mathrm{CHF})$ database is that it has longterm ECG recordings. It consists of 15 subjects with major congestive heart failure.

\section{PROPOSED ALGORITHM}

Data mining is important in healthcare organizations for automating the system. Today in healthcare center a significant amount of data are stored electrically and this stored data is difficult to analyses in the traditional way. So that some algorithms used for prediction described as follows.

\section{A. Deep Learning Methods:}

Deep learning is subfield of machine learning, where machines employ artificial neural network to process information. It consists of various neural networks with a large number of parameters and layers.

1. The speciality of $\mathrm{CNN}$ is that number of layers can be used and each and every layer can be configured manually. These hidden layers provide a great help in predictions where sequence-to-sequence learning is implemented. It can also be used to process data in a variety of forms. It can be image data, text data, numeric data, sound pixels, time-series etc. An example of this model can be InceptionV3, where 48 layers are customized and pre-trained model is used for accurate classification as well as feature extraction.

2. Particle Swarm Optimization (PSO) is used which searches CNN architectures for classification of various images. It evolves the internal parameters of processing layers. In this technique particles move in group for finding better results.

3. In FFNN, information or input data goes only in one direction. The data flows in forward direction from the input nodes to the output nodes through multiple hidden layers of nodes. The hidden layers can be a single layer or a multiple layers.

4. The SVM algorithm has advantage that it can be configured to be used for classification as well as regression. It has an ability to handle multiple continuous and categorical variables.

5. Long short-term memory networks (LSTM) is a type of RNN and is used for time-series data like ECG image dataset. To compare the performance of LSTM networks with other neural networks such as CNN-LSTM, different LSTM models are prepared in the classification of cardiac disease data.

\section{B. Steps of Deep learning models}

1. Collection of ECG image datasets

2. Pre-processing of ECG Dataset

3. Dataset Classification in Training and Testing Subsets.

4. Trial and Error method to carry out experiments and look for best suitable model.

5. Training and testing selected model.

6. Prediction of model.

7. Final Evaluation of model.

In this study, CNN-LSTM model is designed for arrhythmia data analysis which gives better accuracy as compared to other models.

\section{RESULT}

Precision $=($ TP $/$ TP $)+$ FP

Accuracy $($ Recall $)=($ TP $/$ TP $)+$ FN

When R-R detection model is implemented the overall efficiency achieved is $70 \%$. The classification accuracy of ATI-CNN is about $80 \%$. The accuracy of 2D-CNN model is $87 \%$. When 10 -fold cross validation was applied from FFNet approximately $89 \%$ accuracy was examined. $98.2 \%$ of total accuracy was obtained for CNN - LSTM model during performance criteria evaluation. 


\section{International Journal of Engineering Applied Sciences and Technology, 2021 Vol. 5, Issue 11, ISSN No. 2455-2143, Pages 197-200 \\ Published Online March 2021 in IJEAST (http://www.ijeast.com)}

\section{CONCLUSION}

The main motive behind the survey is to find how much accurately we can predict the heart diseases. We can also efficiently diagnose the heart disorder using ECG signals and its developed components. CNN algorithm has been effectively used to identify various cardiac arrhythmias. CNN shows the it's peculiar ability that it can extract the dissimilar features that are relatively invariant to the temporal or local variations. Thus, various deep learning models are used to detect arrhythmia and suggest solutions on some of the important diagnosis. These methods also facilitate decision making in patient management.

\section{REFERENCE}

[1] Yao Qihang, Wang Ruxin, Fan Xiaomao, Li Ye, Liu Jikui, "Multi-class Arrhythmia detection from 12-lead varied-length ECG using Attention-based Time-Incremental Convolutional Neural Network", 2019 ,Science direct, Volume 53

[2] Tun Zin Mar, Khine May Aye," Cardiac Diagnosis Classification Using Deep Learning Pipeline on Apache Spark", IEEE International Conference 2020, 10.1109/ECTICON49241.2020.9158314

[3] Savalia Shalin, Emamian Vahid," Cardiac Arrhythmia Classification by Multi-Layer Perceptron and Convolution Neural Networks", Bioengineering 2018, 5, 35; doi:10.3390/bioengineering5020035

[4] Mazaheri, V., Khodadadi, H., Heart Arrhythmia Diagnosis based on the Combination of Morphological, Frequency and Nonlinear Features of ECG Signals and Metaheuristic Feature Selection Algorithm, Expert Systems with Applications (2020), doi: https://doi.org/10.1016/j.eswa.2020.113697

[5] Bhurane Ankit, Sharma Manish, Ru San-Tan, U. Rajendra Acharya " An efficient detection of congestive heart failure using frequency localized filter banks for the diagnosis with signals "https://doi.org/10.1016/j.cogsys.2018.12.017

[6] Chang Kuan-cheng, Hsieh Po-Hsin, Wu Mei-Yao, Wang Yu-Chen, Chen Jan-Yow, Tsai Fuu-Jen, S.C.Shih Edward, Hwang Ming-Jing \& huang Tzung-Chi. "Usefulness of machine learning -based detection and classification of Cardiac Arrhythmia with 12-lead ECG diagrams ", 2020

[7] E.G. Heywood, T.M. Drake, J.R.L. Wild, J. Lee, M. Wilson, M.J. Lee, A systematic review of Automated Atrial fibrillation detection using a hybrid CNN -LSTM network on ECG datasets. 2017, 490-498

[8]Mukhopadhyay Sayantan, Biswas Shouik, Roy Anamitra, Dey Nilanjan' "Heart disease-Sinus Arrhythmia Prediction System by neural network using ECG analysis." May-Jun 2012, Vol. 2, Issue 3,pp.2361-2365

[9] Jumbukia Shweta, Dabhi Vipul, Prajapati Harshadkumar "ECG beat classification using machine learning techniques".
2018 Int. J. Biomedical Engineering and Technology,Vol. 26, No. 1

[10] Sahay Shalini, A.K.Wadhawani, Wadhawani Sulochana, Bhadauria Sarita "Detection and classification on ECG Signal through machine learning". 10 August 2019, ISSN:22783075, Volume-8-issue-, DOI: 10.35940/ijitee.J1165.0881019

[11] Swapna G, Soman KP, Vinayakumar R, Automated detection of cardiac arrhythmia using deep learning technique", 2018, ScienceDirect, Swapna G et al. / Procedia Computer Science 132 1192-1201

[12] V. Chouhan and S. Mehta, "ECG Arrhythmia Classification using STFT-based Spectrogram and CNN". vol. 8, pp. 144-153, 2018

[13] Rajkumar. A, Ganesan. M, Lavanya. R "Arrhythmia classification on ECG using Deep Learning", 2019, 5th International Conference on Advanced Computing \& Communication Systems (ICACCS)

[14] A. Vishwa, M. Lal, S. Dixit, and P. Vardwaj, "Clasification of arrhythmic ECG data using machine learning techniques," 2011, Int. J. of Interactive Multimedia and Artificial Intell., vol. 1, no. 4, pp. 68-71,

[15] P. Rajpurkar, A.Y. Hannun, M. Haghpanahi, C. Bourn, A.Y. Ng, Cardiologist-level Arrhythmia Detection with Convolutional Neural Networks, 2017 arXiv preprint arXiv:1707.01836. 